\title{
Estigma do câncer de pulmão: conceito, fatores associados e avaliação'
}

Isanne Carolina Pantaleão Cinta Lima²

Cibele Andruccioli de Mattos Pimenta N. ${ }^{3}$

doi:10.11144/Javeriana.IE17-1.edcp

Como citar: Cintra Lima ICP, Mattos Pimenta CA. Estigma do câncer de pulmão: conceito, fatores associados e avaliação. Investig Enferm. Imagen Desarr. 2015;17(1): 97-112. http://dx.doi.org/10.11144/Javeriana.IE17-1.edcp

1. Articulo original de investigación. Recebido: 3 de abril de 2014. Aceito: 8 de outubro de 2014 .

2. Enfermeira. Mestranda do Programa de Pós-Graduação em Enfermagem em Saúde do Adulto (Proesa) da Escola de Enfermagem, Universidade de São Paulo, Brasil. Correio eletrônico: isanne@usp.br

3. Enfermeira. Professora titular da Escola de Enfermagem, Universidade de São Paulo, Brasil. Correio eletrônico: parpca@usp.br 


\section{Resumo}

Introdução: $\mathrm{O}$ artigo trata-se de uma revisão integrativa da literatura com o objetivo de identificar o "estado da arte" sobre estigma percebido por pacientes com câncer de pulmão e os instrumentos utilizados para essa avaliação. Material e método: A amostra foi composta por 11 artigos que foram localizados através das bases de dados Medical Literature Analysis and Retrieval Sistem on-line (Medline), Cumulative Index to Nursing and Allied Health Literature (CINAHL), PsycINFO e Scopus, utilizando-se combinações entre os descritores stigma AND (lung cancer OR lung neoplasm). A busca foi efetuada entre setembro e outubro de 2013. Resultados: O país com mais publicações foi os Estados Unidos (72,7\%) e não houve nenhum estudo brasileiro. As publicações ocorreram entre 2004 e 2013, com predomínio em 2013 (45,4\%); isso indica a atualidade do tema. Quatro instrumentos foram utilizados na avaliação do estigma, sendo que o Cataldo Lung Cancer Stigma Scale (CLCSS) é o único específico para câncer de pulmão. Os achados mais significativos foram: doentes com câncer de pulmão se sentem estigmatizados independente de ser fumante ou não, mais culpados, envergonhados e angustiados do que pacientes com outros tipos de câncer e há forte associação entre estigma e depressão e prejuízo na qualidade de vida. Conclusão: Os estudos nessa área são recentes, escassos e não há estudo em amostra brasileira.

Palavras chave: estigma social; neoplasias pulmonares; hábito de fumar

\section{Estigma del cáncer de pulmón: concepto, factores asociados y evaluación}

\section{Resumen}

Introducción: Este artículo es una revisión integradora de la literatura, cuyo objetivo es identificar el estado de la cuestión respecto al estigma percibido por los pacientes con cáncer de pulmón y los instrumentos utilizados para esta evaluación. Material y métodos: La muestra estuvo conformada por 11 artículos encontrados por medio de bases de datos de análisis de la literatura médica y recuperación en línea: Medline, Cumulative Index de Enfermería y Salud Aliada Literatura (CINAHL), PsycINFO y Scopus, usando combinaciones de descriptores estigma AND (neoplasia de pulmón OR cáncer de pulmón). La búsqueda se realizó entre septiembre y octubre de 2013. Resultados: El país con mayor número de publicaciones fue Estados Unidos (72,7\%), y no hubo ningún estudio brasileño. Las publicaciones se produjeron entre $2004 \mathrm{y}$ 2013, sobre todo en 2013 (45,4\%), lo que indica la importancia del tema. Se utilizaron cuatro instrumentos para evaluar el estigma, siendo el Cataldo Lung Cancer Stigma Scale (CLCSS) el único específico para el cáncer de pulmón. Los hallazgos más significativos fueron: los pacientes con cáncer de pulmón se sienten estigmatizados, independientemente de ser un fumador o no, culpables, avergonzados y ansiosos que otros pacientes con otros tipos de cáncer y hay una fuerte asociación entre el estigma y la depresión y el deterioro de la calidad de vida. Conclusión: Los estudios en este campo son recientes, muy pocos y ningún estudio en una muestra brasileña.

Palabras clave: estigma social; neoplasias pulmonares; hábito de fumar 


\section{Lung Cancer Stigma: concept, assessment and associated factors}

\section{Abstract}

Introduction: This article is an integrative review of the literature, which aims to identify the status of the issue of stigma perceived by patients with lung cancer and the instruments used for this evaluation. Methods: The sample consisted of 11 items found through database analysis of the medical literature and online retrieval: Medline, Cumulative Index to Nursing and significance level (CINAHL), PsycINFO and Scopus, using combinations of descriptors: stigma AND (lung neoplasm OR lung cancer).The search was conducted between September and October 2013. Results: The country with the highest number of publications was the United States (72.7\%), and there was no Brazilian study. The publications were produced between 2004 and 2013, especially in 2013 (45.4\%), indicating the importance of the topic. Four instruments to assess stigma were used, with the Cataldo Lung Cancer Stigma Scale (CLCSS) the only specific to lung cancer. The most significant findings were: patients with lung cancer feel stigmatized, regardless of being a smoker or not, they feel more guilty, ashamed and anxious than other patients with other cancers and there is a strong association between stigma and depression and impaired quality of life. Conclusion: The studies in this field are recent, few and no study in a Brazilian sample.

Keywords: social stigma; pulmonary neoplasms; smoking 


\section{Introdução}

O câncer de pulmão é uma das neoplasias mais frequentes no mundo. Sua incidência mundial aumenta $2 \%$ ao ano (1), sendo diagnosticados cerca de 1.6 milhões de casos novos e 1.4 milhões de mortes em todo o mundo (2).

No Brasil, o número de casos novos estimados para 2014/2015 é de 16.400 homens e de 10.930 mulheres, que totalizam 27.330 novos casos (1). Estima-se que a sua ocorrência está relacionada ao consumo do tabaco e seus derivados de 80 a $90 \%$ dos casos diagnosticados $(1,3)$.

Apesar do avanço da medicina, pouco sucesso houve em relação à cura da doença, sendo o tumor com maior taxa de mortalidade no Brasil entre indivíduos do sexo masculino e o segundo tumor com maior taxa de mortalidade entre as mulheres (4).

As evidências epidemiológicas e experimentais comprovaram uma relação causal entre tabagismo e câncer de pulmão (5), portanto a prevenção e a suspensão do tabagismo são as principais medidas para reduzir a incidência do câncer de pulmão (6).

Para essa conscientização, a partir da década de 70 tornaram mais evidentes as manifestações organizadas para o controle do tabagismo no cenário brasileiro (4).

Embora movimentos como esse tenham sido importante para o controle do tabagismo, as intensas divulgações favoreceram a vinculação entre fumo e doença e a percepção pública do tabagismo com uma escolha de comportamento $(7,8)$.

Com isso, pacientes com câncer de pulmão, sobretudo os fumantes, passaram a ser vistos como responsáveis ou mesmo merecedores da doença, estabelecendo o estigma do câncer de pulmão (9-11). As definições para o conceito estigma variam por se tratar de um fenômeno complexo e aplicável a diferentes contextos $(12,13)$.

Goffman (14) define estigma como um atributo que confere profundo descrédito, um estereótipo visto negativamente pela sociedade. É uma situação na qual o indivíduo não possui a aceitação social plena (15).

O estigma apresenta três tipos diferentes (15): o primeiro diz respeito as abominações do corpo (deformidades físicas). O segundo está relacionado com as culpas de caráter individual, que podem ser representadas por distúrbio mental, vício, alcoolismo, homossexualismo, desemprego e comportamento político radical. E, por último, há os estigmas de raça, nação e religião.

Quando relacionado à saúde, o estigma é definido como uma experiência pessoal caracterizada por exclusão, rejeição, culpa ou desvalorização, que resulta da antecipação de um julgamento adverso sobre um individuo ou um grupo com algum problema de saúde (16).

De acordo com diversos estudos, os pacientes com câncer de pulmão apresentam altos níveis de sofrimento psíquico (17-21) e físico (22) comparada aos pacientes com outros tipos de câncer, qualidade do sono prejudicada (23), comportamento de risco (24), baixa qualidade de vida $(25,26)$ e taxas elevadas de sintomas $(27,28)$. 
Estudo comprova que o estigma e a auto-culpa relacionadas ao câncer de pulmão ainda influenciam no tempo por procura de ajuda médica (29). O estigma do câncer de pulmão também é uma importante barreira na comunicação entre paciente e profissional de saúde (30). Além disso, os pacientes com câncer de pulmão tendem a ser menos encaminhado para o oncologista pelos seus clínicos do que pacientes com outros tipos de câncer (31).

Além do obstáculo citado acima, os cuidadores que acreditam ser a doença culpa do paciente, podem possuir menos empatia e prestar um cuidado frágil ao portador de câncer de pulmão (32), e apresentar sintomas depressivos assim como os pacientes (33).

São poucos os estudos (34) cujo foco principal seja a avaliação da influência do estigma em pacientes com câncer de pulmão, sendo que nenhum estudo brasileiro foi localizado sobre o assunto. Dessa forma, o propósito desse estudo é realizar uma revisão de literatura para identificar as publicações sobre estigma em pacientes com câncer de pulmão, seus conceitos, impactos e fatores associados, além dos instrumentos utilizados para avaliação desse estigma.

\section{Metódo}

Trata-se de uma revisão integrativa, um método que permite agregar estudos primários ou secundários com metodologias distintas, e posteriormente sintetizar as evidências e as conclusões gerais sobre o assunto investigado (35).

Para a construção da revisão integrativa é indispensável percorrer as seis etapas seguintes: identificação da questão de pesquisa, estabelecimento de critérios para inclusão e exclusão de estudos, definição das informações a serem extraídas dos estudos selecionados, avaliação dos estudos incluídos na revisão integrativa, tabela sintese dos estudos, interpretação dos resultados e síntese do conhecimento $(35,36)$.

A revisão originou da seguinte questão norteadora: Qual o estado da arte sobre avaliação do estigma em pacientes com câncer de pulmão?

Os critérios de inclusão dos estudos foram: textos completos que tratavam de estigma em pacientes com câncer de pulmão, nos idiomas inglês, português ou espanhol, publicados em periódicos científicos na área da saúde. Foram excluídas as cartas, editoriais, revisões, teses e dissertações e os artigos que tratavam o estigma sob a percepção de profissionais de saúde e estudantes. A busca foi realizada entre setembro e outubro de 2013 nas bases de dados, Medical Literature Analysis and Retrieval Sistem Online (Medline), especificamente Pubmed; Literatura Latino-Americana e do Caribe em Ciências da Saúde (LILACS); Cumulative Index to Nursing and Allied Health Literature (CINAHL); PsycINFO, e Scopus. A estratégia de busca utilizada foi combinações entre as palavras chave ou descritores no Descritores em Ciências de Saúde (DeCS) e Medical Subject Headings (MeSH): stigma AND (lung cancer or lung neoplasm). 
Nesta busca foram identificados 147 estudos: 38 no PubMed, 29 artigos no CINAHL, 60 no Scopus e 20 artigos na PsycINFO. Não foram localizados estudos na LILACS. Após avaliação das publicações repetidas, restaram 110 artigos. Através da leitura dos títulos e resumos identificaram-se 11 artigos que atendiam os critérios de inclusão, previamente estabelecidos, sendo que todos foram avaliados na íntegra e incluídos nessa revisão.

Para a análise e síntese dos artigos foi desenvolvido um formulário para coleta dos dados de cada artigo incluído na amostra final. As informações que continham nesse formulário eram referentes aos dados da publicação (autores, ano de publicação e país), conceituação de estigma, objetivos, tipo de estudo, características da amostra, instrumento de avaliação do estigma e os resultados. Os achados foram apresentados de maneira descritiva e divididos em três áreas de discussão: conceituação do estigma, fatores que contribuem para o estigma e suas conseqüências e instrumentos de avaliação utilizados.

A partir da análise dos estudos, observou-se que os artigos selecionados foram publicados em periódicos de circulação internacional e na língua inglesa. O país predominante das publicações foram os Estados Unidos (72,7\%), seguido por Reino Unido (18,1\%) e Canadá (9\%). A maioria dos estudos foi publicada no periódico Psycho-Oncology (27,2\%) e em 2013 $(45,4 \%)$. Em relação ao delineamento das pesquisas, sete artigos tiveram abordagem quantitativa, sendo seis estudos do tipo transversais $(54,5 \%)$, e um estudo foi uma coorte prospectiva (9\%). Quatro artigos (36,6\%) foram de natureza qualitativa e utilizaram da técnica de entrevista, entrevista semi-estruturada e a combinação entre entrevista semi-estruturada, análise do discurso individual e grupo focal.

Os objetivos dos estudos permearam a intenção dos pesquisadores em conhecer as percepções, experiências (37) e temas chave (38) do estigma em pacientes com câncer de pulmão, o nível de vergonha, culpa, depressão e ansiedade (39) desses pacientes, as relações entre estigmatização e auto-culpa (40), os fatores que influenciam no atraso do relato dos sintomas (41), as relações entre estigma, angústia e bem estar (42), a associação entre estigma percebido e depressão (43), desenvolver um instrumento para medir o estigma percebido (44), comparar os niveis de estigma de pacientes fumantes e que nunca fumaram, bem como a associação do estigma com depressão e qualidade de vida (45) e elucidar a experiência e enfrentamento de mulheres em relação às crenças sobre o câncer de pulmão (46) e investigar a relação entre estigma, ansiedade, depressão e a gravidade dos sintomas físicos (47).

\section{A análise dos resultados}

Por meio do que foi descrito percebe-se a riqueza de questões de investigação que cercam o tema. Pela tabela 1, observa-se que a média de pacientes com câncer de pulmão envolvidos nos estudos quantitativos foi 130,8, e nos estudos qualitativos foi 34,5. Nota-se também que o instrumento de avaliação mais utilizado foi o Cataldo Lung Cancer Stigma Scale (CLCSS). Síntese dos estudos que compuseram a amostra está apresentada na tabela 1.

TABELA 1. Sintese dos artigos sobre estigma em câncer de pulmão 
selecionados na revisão integrativa (São Paulo, 2013)

\begin{tabular}{|c|c|c|c|c|}
\hline $\begin{array}{l}\text { Autor(es)/ } \\
\text { ano/local }\end{array}$ & $\begin{array}{c}\text { Conceituação de } \\
\text { estigma }\end{array}$ & Objetivo & $\begin{array}{l}\text { Métodos/ins- } \\
\text { trumentos }\end{array}$ & Resultados \\
\hline $\begin{array}{l}\text { Chapple, } \\
\text { 2004, Reino } \\
\text { Unido }\end{array}$ & $\begin{array}{l}\text { É quando a socie- } \\
\text { dade rotula alguém } \\
\text { como contaminado, } \\
\text { menos desejável ou } \\
\text { deficiente. }\end{array}$ & $\begin{array}{l}\text { Entrevistar } \\
\text { pacientes com } \\
\text { câncer de } \\
\text { pulmão (CP) e } \\
\text { explorar suas } \\
\text { percepções e } \\
\text { experiências de } \\
\text { estigma. } \\
\end{array}$ & $\begin{array}{l}\text { Estudo quali- } \\
\text { tativo com } 45 \\
\text { pacientes com } \\
\text { CP. } \\
\text { Entrevista } \\
\text { aberta. }\end{array}$ & $\begin{array}{l}\text { Os participantes se } \\
\text { sentiram estigmatiza- } \\
\text { dos porque a doença é } \\
\text { fortemente associada } \\
\text { ao tabagismo, indepen- } \\
\text { dente do portador ser } \\
\text { fumante ou não. }\end{array}$ \\
\hline $\begin{array}{l}\text { Tod AM, } \\
\text { Craven J, } \\
\text { Allmark P., } \\
\text { 2008, Reino } \\
\text { Unido }\end{array}$ & Não foi conceituado & $\begin{array}{l}\text { Identificar os } \\
\text { fatores que } \\
\text { influenciam no } \\
\text { atraso do relato } \\
\text { dos sintomas } \\
\text { no CP. }\end{array}$ & $\begin{array}{l}\text { Estudo qualita- } \\
\text { tivo } 20 \text { pacien- } \\
\text { tes com CP. } \\
\text { Entrevista semi } \\
\text {-estruturada. }\end{array}$ & $\begin{array}{l}\text { Os fatores foram falta } \\
\text { de conhecimento, } \\
\text { medo, culpa e o estig- } \\
\text { ma devido à associa- } \\
\text { ção com o tabagismo, } \\
\text { fatores culturais e } \\
\text { utilização de modelos } \\
\text { não padronizados de } \\
\text { cuidado a saúde. }\end{array}$ \\
\hline $\begin{array}{l}\text { LoConte et } \\
\text { al., 2008, } \\
\text { EUA }\end{array}$ & $\begin{array}{l}\text { Algo que prejudi- } \\
\text { ca a auto-estima, } \\
\text { a socialização e o } \\
\text { comportamento de } \\
\text { busca pelo cuidado } \\
\text { da saúde. }\end{array}$ & $\begin{array}{l}\text { Avaliar o nível } \\
\text { de vergonha, } \\
\text { culpa, depres- } \\
\text { são e ansiedade } \\
\text { entre pacientes } \\
\text { com CP, de } \\
\text { mama e prós- } \\
\text { tata. }\end{array}$ & $\begin{array}{l}\text { Coorte pros- } \\
\text { pectiva de } 172 \\
\text { pacientes ( } 96 \\
\text { com CP, } 30 \text { com } \\
\text { câncer mama e } \\
46 \text { com câncer } \\
\text { de próstata) } \\
\text { Perceived } \\
\text { Cancer-Related } \\
\text { Stigma. }\end{array}$ & $\begin{array}{l}\text { Houve maior percepção } \\
\text { de estigma em pacien- } \\
\text { tes com CP }(\mathrm{p}<0,01) \\
\text { comparado aos outros } \\
\text { tumores. } \\
\text { Observou-se correla- } \\
\text { ção entre história de } \\
\text { tabagismo e aumento } \\
\text { dos niveis de culpa e } \\
\text { vergonha, independen- } \\
\text { te do tipo de tumor }(\mathrm{r}= \\
0,75 ; \mathrm{p}<0,0001) . \\
\end{array}$ \\
\hline $\begin{array}{l}\text { Else-Quest } \\
\text { NM et al., } \\
2009 \text {, EUA }\end{array}$ & $\begin{array}{l}\text { A pessoa estigmati- } \\
\text { zada é, em nossas } \\
\text { mentes, reduzida } \\
\text { de uma pessoa } \\
\text { inteira e normal a } \\
\text { uma contaminada, } \\
\text { deficiente. }\end{array}$ & \begin{tabular}{|l} 
Examinar as \\
relações entre a \\
estigmatização, \\
auto-culpa e \\
ajuste emocio- \\
nal em pacien- \\
tes com CP, \\
de mama e de \\
próstata. \\
\end{tabular} & $\begin{array}{l}\text { Estudo trans- } \\
\text { versal com } 172 \\
\text { pacientes ( } 96 \\
\text { com CP, } 30 \text { com } \\
\text { câncer mama e } \\
46 \text { com câncer } \\
\text { de próstata). } \\
\text { Perceived stig- } \\
\text { ma }\end{array}$ & $\begin{array}{l}\text { A auto-culpa mediou a } \\
\text { relação entre estigma } \\
\text { e ajustamento psico- } \\
\text { lógico. Os pacientes } \\
\text { com CP tinham maior } \\
\text { probabilidade auto cul- } \\
\text { par-se do que pacientes } \\
\text { com câncer de mama } \\
\text { ou próstata p < 0,01. } \\
\end{array}$ \\
\hline $\begin{array}{l}\text { Cataldo, JK } \\
\text { et al., 2011, } \\
\text { EUA. }\end{array}$ & $\begin{array}{l}\text { Experiência pessoal } \\
\text { caracterizada por } \\
\text { exclusão, rejeição, } \\
\text { culpa ou desvalori- } \\
\text { zação, decorrentes } \\
\text { da antecipação de } \\
\text { um julgamento } \\
\text { adverso. }\end{array}$ & $\begin{array}{l}\text { Desenvolver um } \\
\text { instrumento } \\
\text { para medir o es- } \\
\text { tigma percebido } \\
\text { por pessoas } \\
\text { com CP baseado } \\
\text { na Escala de } \\
\text { Estigma de HIV. }\end{array}$ & $\begin{array}{l}\text { Estudo trans- } \\
\text { versal com } 186 \\
\text { pacientes CP. } \\
\text { Cataldo Lung } \\
\text { Cancer Stigma } \\
\text { Scale. }\end{array}$ & $\begin{array}{l}\text { O instrumento possui } \\
\text { quatro fatores: estigma } \\
\text { e vergonha, isolamento } \\
\text { social, discriminação } \\
\text { e tabagismo. Há boa } \\
\text { confiabilidade -alfa de } \\
\text { Cronbach -0,96. }\end{array}$ \\
\hline
\end{tabular}




\begin{tabular}{|c|c|c|c|c|}
\hline $\begin{array}{l}\text { Autor(es)/ } \\
\text { ano/local }\end{array}$ & $\begin{array}{c}\text { Conceituação de } \\
\text { estigma }\end{array}$ & Objetivo & $\begin{array}{l}\text { Métodos/ins- } \\
\text { trumentos }\end{array}$ & Resultados \\
\hline $\begin{array}{l}\text { Cataldo, JK, } \\
\text { Jahan TM, } \\
\text { Pongquan } \\
\text { VL, 2012, } \\
\text { EUA. }\end{array}$ & \begin{tabular}{|l|} 
Refere-se à anteci- \\
pação ou medo da \\
discriminação e de \\
atitudes negativas e \\
ações relacionadas \\
a uma condição \\
específica. \\
Estigma relacionado \\
Saúde (ERS) é uma \\
experiência pessoal \\
caracterizada pela \\
exclusão, rejeição, \\
culpa, ou desvalori- \\
zação, decorrentes \\
da antecipação de \\
um julgamento des- \\
favorável.
\end{tabular} & $\begin{array}{l}\text { Comparar os } \\
\text { niveis de estig- } \\
\text { ma em pacien- } \\
\text { tes com CP e } \\
\text { a associação } \\
\text { entre estigma, } \\
\text { depressão e QV } \\
\text { de fumantes e } \\
\text { não fumantes. }\end{array}$ & $\begin{array}{l}\text { Estudo trans- } \\
\text { versal com } 192 \\
\text { participantes } \\
\text { com CP. } \\
\text { Cataldo Lung } \\
\text { Cancer Stigma } \\
\text { Scale }\end{array}$ & $\begin{array}{l}\text { Fortes associações en- } \\
\text { tre estigma em pacien- } \\
\text { te com CP e depressão } \\
(\mathrm{r}=0,68 ; \mathrm{p}<0,001) \mathrm{e} \\
\text { qualidade de vida ( } \mathrm{r}= \\
-0,65 ; \mathrm{p}<0,001) \text {. } \\
\text { Não houve diferença } \\
\text { significativa entre fu- } \\
\text { mantes e não fuman- } \\
\text { tes. }\end{array}$ \\
\hline $\begin{array}{l}\text { Gonzalez BD, } \\
\text { Jacobsen PB, } \\
\text { 2012, EUA. }\end{array}$ & \begin{tabular}{|l|} 
Ocorre quando a \\
sociedade rotula \\
uma pessoa e este \\
indivíduo passa a \\
ser submetido a res- \\
postas sociais uni- \\
formes - Modified \\
Labeling Theory.
\end{tabular} & $\begin{array}{l}\text { Avaliar a asso- } \\
\text { ciação entre es- } \\
\text { tigma percebido } \\
\text { e depressão }\end{array}$ & $\begin{array}{l}\text { Estudo trans- } \\
\text { versal com } 95 \\
\text { participantes } \\
\text { com CP em } \\
\text { tratamento qui- } \\
\text { mioterápico. } \\
\\
\text { Social Impact } \\
\text { Scale. } \\
\end{array}$ & $\begin{array}{l}\text { Houve associação posi- } \\
\text { tiva entre a percepção } \\
\text { do estigma e a sinto- } \\
\text { matologia depressiva ( } \mathrm{r} \\
=0,46 ; \mathrm{p}<0,001)\end{array}$ \\
\hline $\begin{array}{l}\text { Lebel, 2013, } \\
\text { Canadá. }\end{array}$ & \begin{tabular}{|l|} 
Ocorre quando a so- \\
ciedade rotula uma \\
pessoa como menos \\
desejável do que \\
os outros ou com \\
deficiência. Estigma \\
sentido refere-se a \\
um sentimento de \\
vergonha internali- \\
zado sobre ter uma \\
condição e um medo \\
da discriminação \\
devido à inferiori- \\
dade imputada ou \\
inaceitabilidade \\
social. \\
Estigma promulga- \\
do refere-se a dis- \\
criminação quando \\
realmente ocorre. \\
\end{tabular} & $\begin{array}{l}\text { Investigar as } \\
\text { relações entre } \\
\text { estigma, angús- } \\
\text { tia e bem estar } \\
\text { em pacientes } \\
\text { com câncer de } \\
\text { cabeça e pesco- } \\
\text { ço e de pulmão. }\end{array}$ & \begin{tabular}{|l|} 
Estudo trans- \\
versal com 206 \\
participantes \\
(107 com CP e \\
99 com câncer \\
de cabeça e \\
pescoço). \\
\\
Subescala de \\
13 itens do Ex- \\
planatory Model \\
Interview Cata- \\
logue (EMIC).
\end{tabular} & $\begin{array}{l}\text { Estigma correlaciona- } \\
\text { se positivamente com } \\
\text { angústia e negativa- } \\
\text { mente com bem-estar. } \\
\text { Pessoas que vivem com } \\
\text { CP relataram mais } \\
\text { estigma do que aqueles } \\
\text { com câncer de cabeça e } \\
\text { pescoço. }\end{array}$ \\
\hline
\end{tabular}




\begin{tabular}{|c|c|c|c|c|}
\hline $\begin{array}{l}\text { Autor(es)/ } \\
\text { ano/local }\end{array}$ & $\begin{array}{c}\text { Conceituação de } \\
\text { estigma }\end{array}$ & Objetivo & $\begin{array}{l}\text { Métodos/ins- } \\
\text { trumentos }\end{array}$ & Resultados \\
\hline $\begin{array}{l}\text { Hamann AH } \\
\text { et al. } 2013 \text {, } \\
\text { EUA. }\end{array}$ & $\begin{array}{l}\text { Um atributo com } \\
\text { profundo descré- } \\
\text { dito. } \\
\text { Uma marca que } \\
\text { liga uma pessoa a } \\
\text { estereótipos indese- } \\
\text { jáveis. }\end{array}$ & $\begin{array}{l}\text { Identificar } \\
\text { temas-chaves } \\
\text { relacionados } \\
\text { com o estigma } \\
\text { em pacientes } \\
\text { com CP. }\end{array}$ & $\begin{array}{l}\text { Estudo quali- } \\
\text { tativo } \\
\text { Entrevistas } \\
\text { semi-estrutura- } \\
\text { das com } 42 \text { pa- } \\
\text { cientes e grupos } \\
\text { focais com } 23 \\
\text { pacientes. }\end{array}$ & $\begin{array}{l}\text { Surgiram duas temáti- } \\
\text { cas: estigma percebido } \\
\text { (sentido) confirmado } \\
\text { entre os fumantes e os } \\
\text { que pararam recen- } \\
\text { temente, e o estigma } \\
\text { internalizado (próprio). } \\
\text { Também foram discu- } \\
\text { tidas as consequências } \\
\text { não adaptáveis (redu- } \\
\text { ção da exposição) e as } \\
\text { adaptáveis (aumento do } \\
\text { apoio) relacionadas ao } \\
\text { estigma. }\end{array}$ \\
\hline $\begin{array}{l}\text { Brown C, } \\
\text { Cataldo J. } \\
\text { 2013, EUA. }\end{array}$ & $\begin{array}{l}\text { Identidade de- } \\
\text { teriorada de um } \\
\text { indivíduo ligada } \\
\text { a atributos que } \\
\text { são negativamente } \\
\text { percebidos pela } \\
\text { sociedade. } \\
\text { Consiste em expe- } \\
\text { riências de discri- } \\
\text { minação (estigma } \\
\text { efetivado), ante- } \\
\text { cipação ou medo } \\
\text { de discriminação } \\
\text { (estigma percebido) } \\
\text { ou a aplicação de } \\
\text { estereótipos negati- } \\
\text { vos para si mesmo } \\
\text { (auto-estigma). } \\
\text { Estigma relacionado } \\
\text { à saúde inclui uma } \\
\text { experiência pessoal } \\
\text { caracterizada por } \\
\text { exclusão, rejeição, } \\
\text { culpa e/ou desvalo- } \\
\text { rização relacionada } \\
\text { a um problema de } \\
\text { saúde. }\end{array}$ & $\begin{array}{l}\text { Elucidar a } \\
\text { experiência } \\
\text { de mulheres } \\
\text { sobreviventes } \\
\text { em longo prazo } \\
\text { de câncer de } \\
\text { pulmão e exa- } \\
\text { minar como as } \\
\text { mulheres ade- } \\
\text { rem ou rejeitam } \\
\text { crenças sobre } \\
\text { sua doença. }\end{array}$ & $\begin{array}{l}\text { Estudo qualita- } \\
\text { tivo com } 8 \text { mu- } \\
\text { lheres sobrevi- } \\
\text { ventes em longo } \\
\text { prazo do câncer } \\
\text { de pulmão } \\
\text { Análise do dis- } \\
\text { curso individual } \\
\text { Entrevistas em } \\
\text { grupo. }\end{array}$ & $\begin{array}{l}\text { Os achados incluíam: } \\
\text { o vício do tabaco e } \\
\text { a propaganda como } \\
\text { possíveis precursores } \\
\text { do estigma do câncer } \\
\text { de pulmão; o possível } \\
\text { papel dos especialistas } \\
\text { como potencializadores } \\
\text { do estigma do câncer } \\
\text { de pulmão; rejeição e } \\
\text { aceitação simultânea } \\
\text { do estigma do câncer } \\
\text { de pulmão; controle de } \\
\text { informações através } \\
\text { das atividades de defe- } \\
\text { sa como uma resposta } \\
\text { para atenuar o estigma } \\
\text { do câncer de pulmão. }\end{array}$ \\
\hline $\begin{array}{l}\text { Cataldo JK, } \\
\text { Brodsky JL., } \\
2013 \text {, EUA. }\end{array}$ & $\begin{array}{l}\text { Refere-se à anteci- } \\
\text { pação ou medo da } \\
\text { discriminação e de } \\
\text { atitudes negativas e } \\
\text { ações relacionadas } \\
\text { ao câncer de pul- } \\
\text { mão. Mesma defini- } \\
\text { ção do artigo } 6 .\end{array}$ & $\begin{array}{l}\text { Investigar a } \\
\text { relação entre } \\
\text { estigma, ansie- } \\
\text { dade, depressão } \\
\text { e a gravidade } \\
\text { dos sintomas } \\
\text { físicos. }\end{array}$ & $\begin{array}{l}\text { Estudo trans- } \\
\text { versal com } 144 \\
\text { participantes. } \\
\text { Cataldo Lung } \\
\text { Cancer Stigma } \\
\text { Scale. }\end{array}$ & $\begin{array}{l}\text { Forte associação entre } \\
\text { estigma e ansiedade } \\
(\mathrm{r}=0,413 ; \mathrm{p}<0,001) \\
\text { depressão }(\mathrm{r}=0,559 ; \mathrm{p} \\
<0,001) \text { e sintomas }(\mathrm{r}= \\
0,483 ; \mathrm{p}<0,001) .\end{array}$ \\
\hline
\end{tabular}

CP: câncer de pulmão.

FonTE: elaboração própria 


\section{Conceituação de estigma}

O conceito de estigma esteve presente em todos os artigos, exceto no estudo de Tod (41). Houve artigos que adotaram um e/ou mais conceitos.

A definição elaborada por Goffman (14) foi tomada como referencial pela metade dos artigos $(37,38,40,42,46)$ por ser uma importante contribuição conceitual do tema por um dos principais estudiosos sobre o assunto.

De acordo com esse autor (14), o estigma é definido como um atributo negativo, que rotula uma pessoa como diferente, diminuído, em desvantagem em relação aos outros ou com uma deficiência percebida negativamente pela sociedade. Este conceito foi subdividido em outros dois conceitos: estigma sentido e estigma promulgado. O primeiro refere-se a um sentimento de vergonha internalizado sobre ter uma condição e ao medo da discriminação devido à inferioridade atribuída ou inaceitabilidade social. Já o segundo refere-se à discriminação quando ela efetivamente ocorre.

Os conceitos propostos por outros autores não diferem do proposto por Goffman, mas acrescentem algum detalhe. Dois estudos $(38,43)$ citam as concepções de Link (12). De acordo com esse autor, o estigma ocorre quando elementos rotulados, estereotipados, pertencentes à separação, perda de status, e a discriminação coocorrem em uma situação de poder que permite que esses processos se desdobrem.

Outro conceito que aparece nos artigos (44-47) é o do estigma relacionado à saúde, definido por Weiss e Ramakrishna (16) como uma experiência pessoal caracterizada por exclusão, rejeição, culpa ou a desvalorização decorrente de um julgamento antecipado desfavorável.

A definição proposta por Van Brakel (48) é citada em dois estudos $(45,46)$. Esse autor refere-se ao estigma sendo percebido como uma antecipação ou medo da discriminação e a ciência de atitudes e ações negativas relacionadas a uma condição específica.

\section{Fatores associados ao estigma e seus impactos}

O primeiro estudo documentado sobre o assunto identificou os seguintes fatores como contribuintes para a estigmatização do câncer de pulmão: a associação da doença e tabagismo, a doença entendida como auto-provocada, a elevada taxa de mortalidade e a morte com sofrimento considerável (37).

Nos estudos qualitativos foi observado que o estigma faz parte da experiência do câncer de pulmão devido à generalização de todos os pacientes portadores dessa doença ser fumantes, ter ocasionado a sua doença $(37,41)$ e por esses motivos terem o tratamento negado $(37)$.

Outro achado desses estudos é o atraso no diagnóstico como consequência do estigma. A associação entre tabagismo e o câncer de pulmão foi apontada pelos pacientes como motivo de descrédito nos relatos de sintomas em relação a outros tipos de câncer. A demora em relatar os sintomas do câncer de pulmão se dá pelo medo, pela falta de conhecimento, assim como a culpa e fatores culturais $(37,40)$. Observou-se, ainda, que a participação nos atendimentos em grupos de apoio pode ser dificultada pela vergonha. No entanto, os grupos podem ajudar os doentes a resistirem à estigmatização e serem menos vítimas da auto-culpa (37). 
Os pacientes referiram que as campanhas anti-tabagismo e a imprensa reforçaram o estigma ao enfatizar a morte ao invés do tratamento, e com isso aumentaram as angústias dos doentes $(37,40)$.

O estigma contribui para diminuição nas discussões sobre a doença e pouca participação nas decisões referentes ao tratamento devido à preocupação e auto-culpa sentidas por esses pacientes (38).

Em relação aos estudos quantitativos, LoConte e colaboradores (39) encontraram maior percepção de estigma nos pacientes com câncer de pulmão quando comparado com os outros grupos. A média do escore na mensuração do estigma foi de 1,93 (DP $\pm 0,85$ ), nos doentes com câncer de pulmão, e $1,45(\mathrm{DP} \pm 0,49)$ entre os grupos com câncer de mama e de próstata $(\mathrm{p}<0,01)$.

Nos estudos conduzidos por Cataldo (44) e Cataldo (45), a média do escore foi de 120,31 (DP = 30,36) e de 102,6 (DP = 31), respectivamente, em escala 46 a 184. Em outro estudo de Cataldo (47), a média do escore foi de 75,7 ( $\mathrm{DP}=18,3$; com intervalo possivel de 31-124).

Gonzalez e Jacobsen (43) encontraram associação entre estigma percebido e sintomatologia depressiva. A pontuação média dos participantes foi de 42,90 (DP $=11,87$ ) no instrumento utilizado para avaliar o estigma, cujo escore varia de 0 a 80.

Ainda no estudo de Cataldo (45) foram encontradas fortes associações entre estigma e depressão $(r=0,68 ; p<0,001)$ e estigma e qualidade de vida $(\mathrm{r}=-0,65 ; \mathrm{p}<0,001)$.

Em um dos seus estudos mais recentes, Cataldo e Brodsky (47) detectaram forte associação entre estigma e ansiedade $(r=0,413 ; p<0,001)$, depressão ( $r=0,559 ; \mathrm{p}<0,001)$ e sintomas $(\mathrm{r}=0,483 ; \mathrm{p}<0,001)$.

\section{Instrumentos para avaliação do estigma}

Nos artigos selecionados, identificaram-se quatro instrumentos para avaliação do estigma em pacientes com câncer de pulmão e um instrumento foi predominante.

O Cataldo Lung Cancer Stima Scale (CLCSS) foi utilizado em três estudos $(44,45,47)$. Este instrumento foi construído e validado nos Estados Unidos, sendo o primeiro instrumento específico para avaliar estigma nos pacientes com câncer de pulmão. O desenvolvimento da HIV Stigma Scale foi baseado no modelo conceitual de estigma percebido (49) e foi referência na construção desse instrumento por atribuir a vergonha aos pacientes com câncer de pulmão, assim como aos com HIV, por terem sido "responsáveis" pela sua doença. Ele é composto por 31 itens distribuídos em quatro subescalas que mensuram: estigma e vergonha, isolamento social, discriminação e tabagismo. A faixa de pontuação da escala CLCSS varia de 31-124 (quanto maior a pontuação, maior o estigma).

Em outros dois estudos $(39,40)$ utilizou-se um instrumento denominado Perceived Cancer-Related Stigma, desenvolvido pelos próprios pesquisadores a partir de um grupo de discussão composto por participantes do grupo de apoio ao paciente com câncer de pulmão. É composto por seis itens em uma escala tipo Likert para avaliar auto-culpa relacionada ao 
câncer, abrangendo os sentimentos de vergonha, culpa e constrangimento sobre o próprio câncer (40).

Outro estudo (33) utilizou a Social Impact Scale (SIS) para avaliar o estigma. Esta escala se propõe em avaliar o nivel de estigmatização para pacientes com HIV/AIDS ou câncer através de 24 itens distribuídos em quatro domínios de percepção do estigma: rejeição social, insegurança financeira, vergonha internalizada e isolamento social. A faixa de pontuação da SIS varia de 24 a 96 (quanto maior a pontuação, maior a estigmatização percebida) (50). O resultado observado no estudo que utilizou a SIS foi uma associação positiva entre o estigma e os sintomas depressivos $(r=0,46 ; p$ $<0,001)$.

Lebel e colaboradores (42) utilizaram uma subescala (13 item) do Explanatory Model Interview Catalogue (EMIC). A EMIC é uma entrevista semi-estruturada que aborda o estigma dentro de conceitos sobre o impacto social da doença. A escala mede os seguintes aspectos do estigma: preocupações em relação à divulgação da doença, sensação diminuída de identidade, e preocupações com a rejeição social e da família. A pontuação varia entre 13 e 52 pontos e não possui ponto de corte (quanto maior a pontuação, maior o estigma) (42).

A média de pontuação dos participantes nesse estudo (42) foi de $19,63(\mathrm{DP}=5,49)$. Os principais resultados encontrados foram uma correlação positiva entre estigma e angústia $(§=0,45 ; p<0,001)$ e correlação negativa entre estigma e bem-estar $(囚=-0,42 ; p<0,001)$. Os doentes com câncer de pulmão relataram mais estigma do que aqueles com câncer de cabeça e pescoço.

\section{Conclusões}

- Os conceitos sobre estigma que predominaram foram os definidos por Goffman, que propõe ser o estigma um rótulo imposto a um individuo visto negativamente pela sociedade.

- Os estudos explicitaram ser o estigma parte da experiência de pacientes com câncer de pulmão tendo como fatores associados a forte relação com o tabagismo, e como este influenciou no atraso do diagnóstico, quer pelo doente que demora a procurar ajuda ou pela demora dos profissionais em valorizar queixas que se relacionem a outros cânceres, que não de pulmão, quando o paciente tem história de tabagismo, além da sintomatologia depressiva, baixa qualidade de vida e bem-estar, altos niveis de culpa, vergonha, angústia e sintomas.

- Todos os instrumentos utilizados foram adaptados a partir de outras doenças (42-44). Embora o CLCSS tenha sido baseado em um instrumento utilizado em pacientes com HIV, esse foi o único instrumento validado especificamente para câncer de pulmão (44). Entretanto, poucos estudos foram realizados com esse instrumento devido a sua criação recente. Além disso, não há nenhum instrumento para avaliar estigma em câncer de pulmão traduzido e validado para a lingua portuguesa. 


\section{Conflito de interesses}

Os autores declaram que não há conflito

\section{Recomendações}

São poucos os instrumentos que avaliam o estigma nos pacientes com câncer de pulmão e nenhum deles foi validado para a língua portuguesa brasileira. Assim, é fundamental a validação ou construção de instrumentos que possibilitem identificar o estigma nos pacientes brasileiros com câncer de pulmão e seus familiares para futuras intervenções.

A temática em questão é atual e possui grande potencial de investigação a ser explorado no mundo e principalmente no Brasil, visando compreender melhor o estigma e os fatores a ele associados, e desenvolver ações de cuidado que contribuam para uma assistência melhor e mais humanizada, o que justifica a realização da presente pesquisa.

\section{Referências}

1. Ministério da Saúde do Brasil, Instituto Nacional de Câncer. Estimativa 2014: incidência de câncer no Brasil. Rio de Janeiro: INCA; 2014.

2. Bray F, Ren JS, Masuyer E, Ferlay J. Estimates of global cancer prevalence for 27 sites in the adult population in 2008. Int J Cancer. 2012;132(5):1133-45.

3. American Cancer Society. cancer facts \& figures 2012. Atlanta: American Cancer Society; 2012.

4. Ministério da Saúde do Brasil, Instituto Nacional de Câncer. Abordagem e tratamento do fumante: consenso 2001. Rio de Janeiro: INCA; 2001.

5. Cornfield J, Haenszel W, Hammond EC, Lilienfeld AM, Shimkin MB, Wynder EL. Smoking and lung cancer: recent evidence and a discussion of some questions. Int J Epidemiol. 2009;38(5):1175-91.

6. Cooley ME, Kaiser LR, Abrahm JL, Giarelli A. The silent epidemic: tobacco and the evolution of lung cancer and its treatment. Cancer Invest. 2001;19(7):739-51.

7. Bayer R, Stuber J. Tobacco control, stigma, and public health: Rethinking the relations. Am J Public Health. 2006;96:47-50.

8. Stuber J, Galea S, Link BG. Smoking and the emergence of a stigmatized social status. Soc Sci Med. 2008;67(3):420-30.

9. Conlon A, Gilbert D, Jones B. Stacked stigma: oncology social worker's perceptions of the lung cancer experience. J Psychosoc Oncol. 2010;28(1):98-115.

10. Lehto RH. Patient views on smoking, lung cancer, and stigma: A focus group perspective. Eur J Oncol Nurs. 2014;18(3):316-22 .

11. Mosher, CE, Danoff-Burg S. An attributional analysis of gender and cancer-related stigma. Sex Roles. 2008;59:827-38.

12. Link BG, Phelan JC. Conceptualizing stigma. Annu Rev Sociol. 2001;27:363-85.

13. Rüsch N, Angermeyer MC, Corrigan PW. Mental illness stigma: concepts, consequences, and initiatives to reduce stigma. Eur Psychiatry. 2005;20:529-39. 
14. Goffman E. Stigma. Englewwodk Cliffs, NJ: Prentice Hall; 1963.

15. Goffman E. Estigma: notas sobre a manipulação da identidade deteriorada. 4a ed. Rio de Janeiro: LTC; 1988.

16. Weiss M, Ramakrishna J. Stigma interventions and research for international health. Lancet. 2006;367:536-8.

17. Zabora J, Brintzenhofesoc K, Curbow B, Hooker C, Piantadosi S. The prevalence of psychological distress by cancer site. Psyco-Oncology. 2001;10:19-28.

18. Sigimura H, Yang P. Long-term survivorship in lung cancer: A review. Chest. 2006;129:1088-97.

19. Lynch J, Goodhart F, Saunders Y, O'Connor SJ. Screening for psychological distress in patients with lung cancer: Results of a clinical audit evaluating the use of the patient distress thermometer. Support Care Cancer. 2010;19:193-202.

20. Domingues V, Albuquerque E. Cancro do pulmão: aspectos psicológicos e psiquiátricos. Rev Port Pneumol. 2008;14(2):261-9.

21. Hewitt M, Rowland JH, Yancik R. Cancer suvivors in the United States: age, health, and disability. J Gerontol A Biol Sci Med Sci. 2003;58:82-91.

22. Kotajima F, Kobayashi K, Sakaguchi H, Nemoto M. Lung cancer patients frequently visit the emergency room for cancer-related andunrelated issues. Mol Clin Oncol. 2014;2(2):322-6.

23. Gooneratne NS, Dean GE, Rogers AE, Nkwuo JE, Coyne JC, Kaiser LR. Sleep and quality of life in long-term lung cancer survivors. Lung Cancer. 2007;58(3):403-10.

24. Evangelista LS, L Sarna, ML Brecht, G Padilla and J Chen. Health perceptions and risk behaviors of lung cancer survivors. Heart Lung. 2003;32:131-9.

25. Sarna L, Brown JK, Cooley ME, Williams RD, Chernecky C, Padill $\mathrm{G}$, et. al. Quality of life and meaning of ilness of women with lung cancer. Oncol Nurs Forum. 2005;32(1):E9-19.

26. Schag CAC, Ganz PA, Wings DS, Sim MS, Lee JJ. Quality of life in adult survivors of lung, colon and prostate cancer. Qaul Life Res. 1994;3:127-41.

27. Yang P, Cheville AL, Wampfler JA, Garces YI, Jatoi A, Clark MM, et al. Quality of life and symptom burden among long-term lung cancer survivors. J Thorac Oncol. 2012;7:64-70.

28. Chan CW, Chair SY, Chui YY. End of life experience of symptom cluster and their management in Hong Kong Chinese patients with lung cancer who receive palliative radiotherapy. Zhongguo Fei Ai Za Zhi. 2009;12(5):361-8.

29. Bell K, Salmon A. Bowers M, Bell J, McCullough L. Smoking, stigma and tobacco 'denormalization': Further reflections on the use of stigma as a public health tool. Soc Sc Med. 2010;70:795-9. 
30. Nelson JE, Gay EB, Berman AR, Powell CA, Salazar-Schicchi J, Wisnivesky JP. Patientes rate physician communication about lung cancer. Cancer. 2011;117:5212-20.

31. Wassenaar TR, Eickhoff JC, Jarzemsky DR, Smith SS, Larson ML, Shiller JH. Differences in primary care clinicians'approach to nonsmall cell lung cancer patients compared with breast cancer. J Thorac Oncol. 2007;2:722-8.

32. LocBuck MM, McClement SE, McPherson C, Cheang M. Does blaming the patient with lung cancer affect the helping behavior of primary caregivers? Oncol Nurs Forum. 2008;35(4):681-9.

33. Siminoff LA, Wilson-Gernderson M, Backer Jr. S. Depressive symptoms in lung cancer patients and their family caregivers and the influence of family environment. Psychooncology. 2010;19(12):1285-93.

34. Chambers SK, Dunn J, Occhipinti S, Hughes S, Baade P, Sinclair $\mathrm{S}$, et al. A systematic review of the impact of stigma and nihilism on lung cancer outcomes. BMC Cancer. 2012;12:184.

35. Mendes KDS, Silveira RCdCP, Galvão CM. Revisão integrativa: método de pesquisa para incorporação de evidências na saúde e na enfermagem. Texto Contexto Enferm. 2008;17(4):758-64.

36. Whittemore R, Knafl $\mathrm{K}$. The integrative review: updated methodology. J Adv Nurs. 2005;52(5):546-53.

37. Chapple A, Ziebland S, McPherson A. Stigma, shame, and blame experienced by patients with lung cancer: Qualitative study. BMJ. 2004;328(7454):1470.

38. Hanann HA, Ostroff JS, Marks EG, Gerber DE, Schiller JH, Craddock Lee SJC. Stigma among patients with lung cancer: a patient-reported measurement model. Psychooncology. 2014;23(1):81-92.

39. LoConte NK, Else-Quest NM, Eickhoff J, Hyde J, Schiller JH. Assessment of guilt and shame in patients with non-small-cell lung cancer compared with patients with breast and prostate cancer. Clin Lung Cancer. 2008;9(3):171-8.

40. Else-Quest NM, LoConte NK, Schiller JH, Hyde JS. Perceived stigma, self-blame, and adjustment among lung, breast and prostate cancer patients. Psychol Health. 2009;24(8):949-64.

41. Tod AM, Craven J, Allmark P: Diagnostic delay in lung cancer: a qualitative study. J Adv Nurs. 2008;61(3):336-43.

42. Lebel S, Castonguay M, Mackness G, Irish J, Bezjak A, Devins GM. The psychosocial impact of stigma in people with head and neck or lung cancer. Psychooncology. 2013;22(1):140-52.

43. Gonzalez BD, Jacobsen PB. Depression in lung cancer patients: The role of perceived stigma. Psychooncology. 2012;21(3):239-46.

44. Cataldo JK, Slaughter R, Jahan TM, Pongquan VL, Hwang WJ. Measuring stigma in people with lung cancer: psychometric testing of the cataldo lung cancer stigma scale. Oncol Nurs Forum. 2011;38(1):E46-54. 
45. Cataldo JK, Jahan TM, Pongquan VL. Lung cancer stigma, depression, and quality of life among ever and never smokers. Eur J Oncol Nurs. 2012;16(3):264-9.

46. Brown C, Cataldo J. Explorations of lung cancer stigma for female long-terms survivors. Nurs Inq. 2013;20(4):352-62.

47. Cataldo JK, Brodsky JL. Lung cancer stigma, anxiety, depression and symptom severity. Oncology. 2013;85(1):33-40.

48. Van Brakel W. Measuring health-related stigma. A literature review. Psychol Health Med. 2006;11(3):307-34.

49. Berger B, Ferrans C, Lashley F. Measuring stigma in people with HIV: Psychometric assessment of the HIV stigma scale. Res Nurs Health. 2001;24:518-29.

50. Fife BL, Wright ER. The dimensionality of stigma: A comparison of its impact on the self of persons with HIV/AIDS and cancer. J Health Soc Behav. 2000;41:50-67. 